\title{
Interview with Noga Alon
}

Toufik Mansour

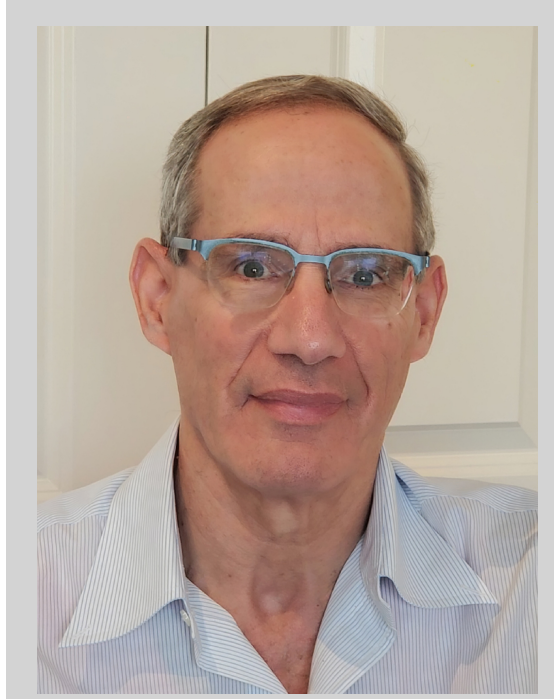

Noga Alon graduated from the Hebrew Reali School in 1974, and received his Ph.D. in Mathematics at the Hebrew University of Jerusalem in 1983. After his Ph.D., he spent the following two years in MIT and then joined Tel Aviv University in 1985. He had visiting positions in various research institutes including MIT, Harvard, the Institute for Advanced Study in Princeton, IBM Almaden Research Center, Bell Labs, Bellcore and Microsoft Research. He is currently a Professor of Mathematics at Princeton University and a Baumritter Professor Emeritus of Mathematics and Computer Science at Tel Aviv University, Israel. He has made significant contributions to combinatorics and theoretical computer science. He has given lectures in many conferences, including plenary addresses in the 1996 European Congress of Mathematics and in the 2002

International Congress of Mathematicians. Alon has received a number of awards, including the Erdös Prize, the Feher Prize, the Pólya Prize, the Bruno Memorial Award, the Landau Prize, the Gödel Prize, the Israel Prize in Mathematics, the EMET Prize in Mathematics, the Dijkstra Prize, the Nerode Prize and the Kanellakis Award. He has been a member of the Israel Academy of Sciences and Humanities since 1997, and is also a member of Academia Europaea and of the Hungarian Academy of Sciences. He is an AMS Fellow, and an ACM Fellow, and serves as the editor-in-chief of Random Structures and Algorithms.

Mansour: Professor Alon, first of all we would like to thank you for accepting this interview. Would you tell us broadly what combinatorics is?

Alon: Combinatorics is primarily the mathematics of finite objects, investigating the properties of combinatorial structures. Its areas of study include exact and asymptotic enumeration, graph theory, probabilistic and extremal combinatorics, designs and finite geometries. The subject has applications in other areas including geometry, number theory, optimization and theoretical computer science.

Mansour: What do you think about the development of the relations between combinatorics and the rest of mathematics?

Alon: The relations between combinatorics and other mathematical and scientific areas have been crucial in the development of the modern theory. Combinatorial concepts and questions appear naturally in many branches of mathematics, and the area has found applications in other disciplines as well. These include applications in information theory and electrical engineering, in statistical physics, in chemistry and molecular biology, and, of course, in computer science. Combinatorial topics such as Ramsey theory, combinatorial set theory, matroid theory, extremal graph theory, combinatorial geometry and discrepancy theory are related to a large part of the mathematical and scientific world, and these topics have found numerous applications in other fields.

Mansour: Why is there a strong relationship

The authors: Released under the CC BY-ND license (International 4.0), Published: October 19, 2020

Toufik Mansour is a professor of mathematics at the University of Haifa, Israel. His email address is tmansour@univ.haifa.ac.il. 
between combinatorics and theoretical computer science?

Alon: The core of most of the fundamental questions in theoretical computer science is combinatorial, as the notion of computation is based on manipulations with finite structures. The investigation of the limits of computation leads to basic combinatorial questions, and much of the design and analysis of efficient algorithms is also combinatorial in nature.

Mansour: What have been some of the main goals of your research?

Alon: In my research I tried (and still try) to develop effective techniques by tackling interesting problems. Solving such problems, especially ones with a history, is an important goal, but an even more important goal is the introduction of novel ideas and tools that can lead to further progress.

Mansour: What were your early experiences with mathematics? Were there specific problems that made you first interested in combinatorics?

Alon: From an early age I have been interested in solving mathematical puzzles; I participated in several mathematics competitions for high school students in Israel and read popular mathematical books. An early encounter with a mathematical proof that impressed me was when I was in high school and first learned about the probabilistic method. I read a version of one of the earliest results established using it: the proof of the lower bound for Ramsey numbers discovered in 1947 by Paul Erdős, the founder of the method. I still remember the admiration I felt going through the concise and elegant argument, an admiration that only increased when I kept following the profound impact of other applications of the method on the development of discrete mathematics.

Mansour: We would like to ask you about your formative years. What was the first reason you become interested in mathematics and specifically combinatorics? Did that happen under the influence of your family, or some other people?

Alon: As already mentioned, I have been interested in mathematical puzzles since I was a child. My parents always encouraged me and my brother to pursue whatever we found appealing, with no special emphasis on exact sciences. During my final two years in high school
I had a superb teacher of mathematics, a new immigrant from Ukraine, who taught us advanced material after the usual hourly classes. In my final year in high school, I also met Paul Erdős who has been visiting Israel frequently, and heard several questions from him that I found fascinating.

Mansour: What was the reason you chose the Hebrew University of Jerusalem for your Ph.D., and your advisor, Micha Asher Perles? Alon: I finished my undergraduate degree at the Technion, M.Sc. at Tel Aviv University, and Ph.D. at the Hebrew University of Jerusalem, hence, I had some experience as a student in all three institutions. However, I completed both the M.Sc. and Ph.D. degrees during my army service in the academic reserve, when I have only been able to come to the university once a week. I first heard about Micha Perles from Meir Katchalski, who taught me at the Technion, and after meeting him, when I was about to finish my undergraduate degree, I became convinced that I want to do my research work with him. Micha has in fact been my M.Sc. and my Ph.D. advisor, and I learned a lot from him, his profound interest in the close connection between combinatorics and geometry, and his general approach to mathematical research.

Mansour: What was the problem you worked on in your thesis?

Alon: In my M.Sc. thesis, I worked on a problem that originated from a discussion I had with Erdős, who suggested a special case. The problem was to determine or estimate the maximum possible number of copies of a fixed graph $H$ in a (simple) graph with a given number of edges. It turned out that the parameter determining the asymptotic behavior of this maximum is the fractional cover (or matching) number of the graph. When I worked on the problem, I was not aware of much of the existing results about this notion, which I redefined, and although I could have saved quite some time had I been more familiar with the known literature, this ignorance did lead to some interesting new discoveries. The results obtained appear in my very first paper. My Ph.D. thesis includes a chapter with an extension of this problem and also deals with additional extremal combinatorial problems.

Mansour: How was the mathematics in Is- 
rael and specifically at the Hebrew University of Jerusalem at the time?

Alon: Israel has traditionally always been a strong center of mathematical research. The Hebrew University has been particularly strong in Set Theory, Logic and Ergodic Theory. As mentioned, I used to come to the university only once a week. We had an active combinatorics seminar with other graduate students and young researchers who are roughly of my generation, including Nati Linial, Gil Kalai, Yaakov Kupitz, Roy Meshulam and Ron Adin. I still have fond memories from this period.

Mansour: Would you discuss for a little bit about your most influential results and why they have been influential?

Alon: The work on expander graphs and their applications, focusing on the tight relation between the spectral properties of a graph and its structural properties, has been influential due to the numerous applications of expanders in theoretical computer science and coding theory and the more recent interest in their study in group theory and number theory.

The investigation of derandomization techniques, that is, techniques for converting randomized algorithms into deterministic ones, including the Color Coding method with Yuster and Zwick that became an important tool in what's called Parameterized Complexity.

The work with Matias and Szegedy is the foundational paper on streaming algorithms which played a major role in creating this active area.

The Combinatorial Nullstellensatz is a powerful algebraic technique with applications in Graph Theory, Combinatorics and Additive Number Theory.

The work on the regularity lemma of Szemerédi, its extensions and applications in property testing, stimulated research on the structure of large graphs and is closely related to the investigation of convergent graph sequences.

The book with Spencer is the leading text on probabilistic methods in combinatorics, which is a very active area in modern combinatorics.

Besides these, there are several papers that settle long standing problems including a problem of Shannon on the zero-error capacity of the disjoint union of two channels, and the
Hadwiger-Debrunner $(p, q)$-problem in Combinatorial Geometry.

Mansour: What would guide you in your research? A general theoretical question or a specific problem?

Alon: I usually like to think about specific problems in the hope that progress in their study will lead to the development of novel methods and provide new insights on more general topics.

Mansour: When you are working on a problem, do you feel that something is true even before you have the proof?

Alon: This varies. Sometimes I am sure I know the correct answer even before I see a proof, and sometimes I don't know whether a statement is true or not and I spend comparable amount of time trying to find a proof and trying to think about counter-examples. During the years, however, it happened to me several times that I was convinced about the correct answer before I knew a proof and this turned out to be wrong. I sometimes found such unexpected counterexamples by myself, while trying to prove the converse, and sometimes learned about such examples by other researchers. I am thus trying to always remind myself that even a strong intuition about the validity of a statement before there is an actual proof should always be taken with a grain of salt, taking into account the possibility that the intuition may be misleading.

Mansour: What three results do you consider the most influential in combinatorics during the last thirty years?

Alon: This is not an easy question, and I can come up with several different choices. Here is one possibility:

The Graph Minors monumental project of Robertson and Seymour.

The Interlacing Polynomials method of Marcus, Spielman and Srivastava which led to the proof of existence of bipartite Ramanujan graphs of all degrees, and to the solution of the Kadison-Singer problem and its variants.

The proof of Keevash that the obvious necessary divisibility conditions for the existence of designs are also sufficient, for all values of the parameters, provided the number of elements of the ground set is sufficiently large, settling asymptotically the main problem in design theory raised by Steiner in the 19th cen- 
tury.

Mansour: What are the top three open questions in your list?

Alon: Here, too, I can give several answers, here is one:

The $\mathrm{P}$ versus NP problem, formulated as a question in Circuit Complexity (whose solution could be even stronger than solving the $\mathrm{P}$ versus NP problem).

Finding a proof of the Four Color Theorem which is not computer-aided, possibly by using algebraic tools.

Deciding if the maximum possible Shannon capacity of a graph with independence number 2 is bounded. Equivalently: deciding if there is a finite constant $\mathrm{C}$ so that the maximum possible number of vertices in a complete graph whose edges can be colored by $k$ colors with no monochromatic triangle is at most $C^{k}$ (this is sometimes called the Schur-Erdős problem). Mansour: What kind of mathematics would you like to see in the next ten-to-twenty years as the continuation of your work?

Alon: I would love to see new applications and further development of my Combinatorial Nullstellensatz, which has already been extended in various directions. It has been used extensively in Combinatorics, Graph theory, and Additive Number Theory, but I believe that additional development may lead to more sophisticated applications. Progress here should probably combine combinatorial reasoning with tools from algebraic geometry.

I have already seen fascinating extensions of some of my other results, like the necklace splitting theorem, the $(p, q)$-theorem in combinatorial geometry and the research of streaming algorithms, and it is always rewarding to see more. I also hope, of course, to play an active role in at least some of these potential future developments.

Mansour: What would you say about some of the major directions in combinatorics for the next two decades?

Alon: The tight connection with theoretical computer science suggests that an algorithmic point of view, and maybe also computer aided proofs, will play a major role in the future developments of the subject. I also believe that modern combinatorics will use even more tools from other branches of mathematics in the future. Applications of combinatorial techniques in Number Theory and Statistical Physics are very active already and are likely to keep developing. I also hope to see directions that are hard to predict at the moment.

Mansour: Do you think that there are core or mainstream areas in mathematics? Are some topics more important than others?

Alon: There are always areas in mathematics that are considered mainstream, but these keep changing. Moreover, some of the most exciting mathematical results combine different areas, and are based on sometimes unexpected relations between seemingly unrelated fields. I thus believe that mathematics should be considered as one unit, and we should not try to identify the important and less important areas.

Mansour: What do you think about the distinction between pure and applied mathematics that some people focus on? Is it meaningful at all in your own case? How do you see the relationship between so-called "pure" and "applied" mathematics?

Alon: In his book, "A Mathematician's Apology", Hardy stresses his opinion that the most beautiful mathematics is pure mathematics, that has no practical applications in the outside world. He gives his own special field, number theory, as an example of a topic that would never have any practical applications. As we know now, Number Theory, prime numbers and algorithms for factorization, play a major role in Cryptography, which is one of the most applicable mathematical areas. This indicates that the border between "pure" and "applied" mathematics is very unclear, and keeps changing. Personally, I like to work on interesting mathematical problems without thinking much about their potential practical applications, if any. But if some of the results do lead to practical applications (as happened to me with the case of streaming algorithms), I view it as an extra merit.

Mansour: What advice would you give to young people thinking about pursuing a research career in mathematics?

Alon: Think about the problems and the areas you find interesting, without trying to identify the "important" ones. The key to success is to be enthusiastic about your subject. In addition, try to learn as much mathematics as you can early. It is much harder to learn new topics 
later, and having a strong general mathematical background is indispensable.

Mansour: Would you tell us about your interests besides mathematics?

Alon: Reading, traveling around the world, playing table tennis (enthusiastically even if not at a very high level).

Mansour: Before we close this interview with one of the foremost experts in combinatorics, we would like to ask some more specific mathematical questions:

You have worked on many problems from combinatorics and theoretical computer science throughout your career. Which one is your favourite?

Alon: The application of basic properties of polynomials in these areas. This includes the construction of small sample spaces supporting $k$-wise independent random variables, which is useful in the derandomization of randomized algorithms. It also includes a convenient way I found for bounding the Shannon capacity of a graph that led to a solution of a problem raised by Shannon in 1956. Notably, it also includes the Combinatorial Nullstellensatz, a variant of Hilbert's fundamental Nullstellensatz which is the basis of Algebraic Geometry. The Combinatorial Nullstellensatz found applications in Combinatorics, Graph Theory and Additive Number Theory. When it works it often gives optimal results and usually hardly requires any tedious computation.

Mansour: Your book with Joel Spencer is the main reference for the probabilistic method. What is the probabilistic method? Is there a result that you consider the most interesting obtained by the probabilistic method? Any comments on the research in this direction?

Alon: The probabilistic method is a powerful technique for proving deterministic statements by showing that a random structure or substructure of a given one in an appropriately defined sample space satisfies a set of desired properties with positive probability, and therefore exists. Although this may sound naive, the power of the technique is that it enables one to apply techniques from probability theory in the study of problems in combinatorics, number theory, geometry and more. One of the first applications of the probabilistic method in combinatorics is the proof of Erdös, the founder of the method, of an exponential lower bound for the diagonal Ramsey numbers: for every integer $t>2$ there are graphs with at least $2^{t / 2}$ vertices that contain neither a clique nor an independent set of size $t$. Although, by now, this short and simple proof appears in the first chapter in any text-book on the subject, its subtlety can be appreciated by the fact that we still do not know any efficient algorithm for constructing such graphs. A more sophisticated result obtained by the method is the existence of graphs with high girth and high chromatic number. The method is one of the most powerful and widely used tools in modern Combinatorics and its applications. I believe and hope that our book contributed to the success and popularity of the subject.

Mansour: How would you describe the extremal combinatorics? What would you suggest for a graduate student and young researcher who wants to work in this field?

Alon: Extremal Combinatorics, in its strictest sense, deals with the problem of determining or estimating the maximum or minimum possible cardinality of a collection of finite objects that satisfies certain requirements. The area is, however, much broader, including in its scope the investigation of inequalities between combinatorial invariants, and questions dealing with relations among them. Extremal combinatorial problems are often related to other areas including Computer Science, Information Theory, Number Theory and Geometry. This branch of Combinatorics has developed spectacularly over the last few decades. A young researcher interested in the field can start by reading some of the books or surveys on the subject. The book of Jukna, extremal combinatorics with applications in computer science, with its many references is one excellent source.

Mansour: What is an expander? Why did they become so popular? Any comments on the research in this direction?

Alon: Expanders are sparse graphs with strong connectivity properties. A formal definition for a family of expanders is an infinite family of $d$-regular graphs, where for each member of the family, every set $U$ of at most half of its neighbors has at least $c|U|$ neighbors outside the set. The crucial fact here is that $d$ and $c>0$ are fixed for all the graphs in the family. Such graphs with various val- 
ues of the parameters have numerous applications, some, like the construction of interconnection networks, are natural given the definition, but some are much less expected and include derandomization techniques, construction of error correcting codes, the design of efficient sorting networks, and more. The reasons for the popularity of the subject are not only the applications but also the fact that the study of the subject led to the introduction of many interesting techniques and to the discovery of tight connections it has to deep results in Number Theory and Group Theory. The area is still very active, with exciting questions about explicit constructions, about the combinatorial pseudo-random properties of expanders and about higher dimensional extensions.

Mansour: In your 1987-paper on "Splitting necklaces" *, you gave a short beautiful proof by using topological results. What do you think about the importance of being competent in different branches of mathematics?

Alon: Many of the most exciting results in Mathematics are obtained by combining tools from seemingly unrelated areas. It is, therefore, crucial to be as knowledgeable as possible in diverse mathematical areas, even if one is interested mainly in applications in a more restricted subject. In my own research, I managed several times to use tools and intuition from other areas in the study of combinatorial problems. Examples include the application of Grothendieck's Inequality in the development of efficient algorithms for large graphs, using character sum estimates in the study of Cayley graphs, relying on the intuition arising from classical isoperimetric inequalities in the study of expanders, applying results in real algebraic geometry including theorems of Milnor and Warren in the investigation of sign rank of matrices and related problems, and the application of topological, algebraic, probabilistic and spectral techniques in discrete mathematics and in theoretical Computer Science. There are, of course, lots of important mathematical areas in which my knowledge is very limited. One reminder I got of this fact was when I served as the chair of the scientific committee of the 2006 international congress of mathematicians. I wish I had a better global knowl- edge of mathematics, I am trying regularly to hear at least some lectures focused on areas in which I am far from being an expert, and believe that it is desirable to learn the basics of different branches of mathematics already at the beginning of a mathematical career.

Mansour: Mathematicians have been working on graphs for centuries and have developed several tools to understand their structures. Suppose someone comes with a "large" graph and asks (naively) your help to understand the structure of her graph. How would you help her? What are the basic tools she can use?

Alon: The regularity lemma of Szemerédi and its variants form a basic tool in understanding the structure of large (dense) graphs. Although the dependence of the statements on the parameters is highly non-practical, the insight the lemma and its applications in what's known as graph property testing provide is useful. Property testing has been investigated intensively for sparse graphs as well, showing that sampling techniques can often provide a lot of information about a large graph, even when we sample only a tiny fraction of it.

Mansour: What kind of enumerative problems look interesting to you? Why is it very difficult to count some combinatorial objects? Do you think that mathematicians need to invent some new tools to count such objects or just use the existing tools in an ingenious way? Alon: It is a fascinating fact that there are problems in enumerative combinatorics that are very simple to state and look extremely hard to solve. Recall that for $n>k$ a permutation $\pi \in S_{n}$ contains a permutation $\sigma \in S_{k}$ if the permutation matrix of $\pi$ contains that of $\sigma$ as a submatrix. If $\pi$ does not contain $\sigma$, it is called $\sigma$-avoiding. What is the number of 1324-avoiding permutations? Does it have a nice recurrence relation, and why is this problem so much more difficult than the well understood case of avoiding a permutation of length 3? What is the number of 132-avoiding cyclic permutations? Is it roughly the total number of 132-avoiding permutations divided by $n$ ? Is the number of integers $n$ between 1 and $N$ for which the partition function $p(n)$ is even $(1 / 2+o(1)) N$ ? To me, personally, the asymptotic problems are often more interesting

*https://www-sciencedirect-com.ezproxy.haifa.ac.il/science/article/pii/0001870887900557?via\%3Dihub 
than the ones dealing with precise counting. A specific (admittedly somewhat special) problem I found (and still find) interesting, motivated by a question about Hamilton cycles in tournaments, is the asymptotic behavior of the number of cyclic permutations $\left(a_{0}, a_{1}, \ldots, a_{2 k}\right)$ of $Z_{2 k+1}$ in which for every $i$ the difference $\left(a_{i+1}-a_{i}\right)$ modulo $2 k+1$ is at most $k$. Is this number much larger than $Q_{k}=(2 k) ! / 2^{2 k+1}$ for large $k$ ? That is, does the ratio between this number and the quantity $Q_{k}$ tend to infinity as $k$ tends to infinity?

Mansour: Would you tell us about your thought process for the proof of one of your most important results? How did you become interested in that problem? How long did it take you to figure out a proof? Did you have a "eureka moment"?

Alon: There are results that are among my best on which I spent a very long time. I have worked on expanders for years, and in fact even recently investigated the delocalization of eigenvectors of high girth expanders. This involved reading a lot, and thinking a lot over the years, discussing the subject with other researchers and returning to it time and again. Similarly, I spent a long time working on different variants of the polynomial method that yielded the combinatorial nullstellensatz (although the basic end result is not complicated). Here, the main results evolved with the investigation of a considerable number of particular problems. There are, however, results that I believe are important in which there has been a very clear and quick "eureka moment". Our paper with Kleitman in which we solved the Hadwiger-Debrunner $(p, q)$-problem raised in the 50s was a result obtained in essentially one day. Kleitman was visiting me in Tel Aviv, we heard a seminar lecture in which someone mentioned the problem (that I did hear before), and we had the main idea in that afternoon. Finishing the proof took a bit longer, but it was clear already in that day that we know how to do it. I had a similar experience with a paper with Rödl settling a Ramsey theoretic problem raised by Erdős and Sós in 1979. This was during an Oberwolfach conference in 2002, someone mentioned this problem during the open problems session, and we came with the key idea almost immediately. Here, too, this was a problem we have both heard before. Naturally, the detailed proof required more work that took a few weeks, but the basic crucial idea was nearly instantaneous. It sometimes happens that you see the right approach for tackling a problem immediately, possibly because you have seen recently something relevant, or thought about a closely related topic, and it may well be the case that in another day and another state of mind you will have no clue considering the very same question. My final example of a quick solution is a paper I have written around 1990, solving a problem of Erdős and Purdy. My colleague Amos Fiat, who is now a well known computer scientist, met me in the corridor of the mathematics building in Tel Aviv University and showed me a book of Richard Guy: Unsolved Problems in Number Theory, Springer, 1981, that he received from his advisor Adi Shamir. I was on my way to a colloquium lecture, so I just took a quick glance at the book, opening it at a random place and reading a problem that looked interesting and easy to state. During the lecture, I figured out an approach that seemed promising for tackling the problem, and later that day, I basically had the full solution. I went back to Amos, showed him the solution and asked to take a closer look at Guy's book, as it seemed likely, based on the single sample I observed, that I should be able to solve a large fraction of the problems in this book. I then spent a week going carefully over the problems in the book, one by one, and had no idea how to make any progress in the investigation of any of them, besides the first one I have initially picked. I don't claim that there is any meaningful moral here, but think it is an interesting (and accurate) story.

Mansour: Is there a specific problem you have been working on for many years? What progress have you made?

Alon: I have been working on questions that deal with expander graphs for many years. It is not difficult to show that random regular graphs are typically very good expanders, much better than the best known expanders we know to construct explicitly. The explicit constructions use the connection between the expansion properties of a regular graph and its second largest eigenvalue, a connection that appears in my work with Milman and in other papers. This approach cannot lead to ex- 
panders that are nearly as good as the random ones, as the spectral approach is limited by a lower bound on the second largest eigenvalue of any large bounded degree regular graph, that I established with Boppana. I (and many others) have been thinking on and off for years about alternative ways to prove expansion, but, at the moment, there is no known approach that gives explicit constructions that are as good as the random ones. Such constructions will have interesting applications in theoretical computer science and other areas.

Mansour: Professor Noga Alon, I would like to thank you for this very interesting interview on behalf of the journal Enumerative Combinatorics and Applications. 